\title{
Lavoisier et la Naissance de la Thermochimie
}

\author{
BERNARD VALEUR*
}

\begin{abstract}
Les expériences de Lavoisier et Laplace utilisant des calorimètres à glace ont permis pour la première fois de mesurer la chaleur dégagée au cours de réactions chimiques, et en particulier la chaleur de formation de l'eau à partir de ses éléments.

La thermochimie est née avec ces expériences. Quant à l'ensemble des travaux de Lavoisier et Laplace qui comportent également des mesures de chaleurs spécifiques et de chaleurs animales, il marque une étape importante dans l'histoire de la science de la chaleur.
\end{abstract}

Livres [1,2], articles [3-5], colloques, expositions et autres manifestations célèbrent dignement cette année le bicentenaire de la mort d'Antoine-Laurent Lavoisier et rendent ainsi hommage à cet homme dont l'oeuvre politique et scientifique est d'une exceptionnelle diversité. C'est évidemment en tant que I'un des pères de la chimie moderne qu'il est le plus connu. Parmi les nombreux aspects de la "révolution chimique" dont il a été l'un des plus actifs participants, celui qui concerne la chaleur et plus particulièrement les effets thermiques associés aux réactions chimiques - c'est à dire ce qu'on appelle aujourd'hui la thermochimie - est particulièrement intéressant à divers titres: originalité des concepts, ingéniosité des appareils conçus, diversité des phénomènes étudiés, méthodologie,

En 1782, Lavoisier sollicite PierreSimon de Laplace pour entreprendre des recherches sur la chaleur. Laplace était un savant déjà réputé pour ses travaux en astronomie, en mathématiques et en physique; il avait déjà travaillé avec Lavoisier depuis 1777 sur la mise au point de baromètres et de dilatomètres. Pourtant, Laplace refuse la proposition de Lavoisier en expliquant qu'il ne se sent ni le courage, ni la volonté d'entreprendre la nécessaire lecture des nombreux ouvrages de physique et de chimie déjà parus et qu'il préfère se consacrer à des travaux de géométrie et d'analyse. II faudra toute la force de persuasion de Lavoisier pour qu'il se laisse convaincre. L'objectif de Lavoisier est de réfléchir à la notion de chaleur, de concevoir des appareils pour la mesure précise de quantités de chaleur et d'entreprendre des séries d'expériences sur la chaleur spécifique des corps, la chaleur dégagée lors de réactions chimiques, la chaleur animale,...

Auparavant, le concept de chaleur latente avait été introduit par Black en 1762: c'est la quantité de chaleur mise en jeu au cours du changement d'état d'un corps. Le nom de "latente" traduit le fait qu'il s'agit d'une quantité de chaleur non détectable par un thermomètre; elle est en quelque sorte "cachée" et peut se libérer lorsque, par exemple, un gaz se condense. Par ailleurs, la définition de la chaleur spécifique résultait de l'observation selon laquelle il faut fournir des quantités de chaleur différentes pour élever d'un degré la température d'une unité de poids de substances différentes. La chaleur spécifique de l'eau fut prise conventionnellement égale à l'unité et celles des autres corps s'en déduisaient par comparaison. Black avait ainsi déterminé les chaleurs spécifiques de divers corps en utilisant la méthode des mélanges pour mesurer les quantités de chaleur. Cette méthode, imaginée par Crawford, reposait sur le fait que la température finale de deux substances de même poids, initialement portées à des températures différentes puis mélangées, dépend de leurs chaleurs spécifiques. Ainsi Black avait-il jeté les premiers fondements de la science de la chaleur qui allait atteindre son apogée 60 ans plus tard avec les célèbres travaux de Sadi Carnot sur la puissance motrice du feu.

\section{LE CONCEPT DE CHALEUR}

Mais qu'est-ce que la chaleur? A l'époque où Lavoisier et Laplace débutèrent leurs travaux, en 1782, deux conceptions différentes faisaient l'objet d'un débat dans la communauté scientifique. Certains considéraient la chaleur comme une substance fluide, d'autres comme l'effet du mouvement des molécules qui constituent les corps. Dans l'article premier de leur célèbre Mémoire sur la Chaleur [6] Lavoisier et Laplace résument avec précision les deux conceptions: "Les physiciens sont partagés sur la nature de la chaleur. Plusieurs d'entre eux la regardent comme un fluide répan$d u$ dans toute la nature, et dont les corps sont plus ou moins pénétrés, à raison de leur température et de leur disposition particulière à le retenir; (...). D'autres physiciens pensent que la chaleur n'est que le résultat des mouvements insensibles des molécules de la matière. On sait que les corps, même les plus denses, sont remplis d'un grand nombre de pores ou de petits vides, dont le volume peut surpasser considérablement celui de la matière qu'ils renferment; ces espaces vides laissent à leurs parties insensibles la liberté d'osciller dans tous les sens, et il est naturel de penser que ces parties sont dans une agitation continuelle (...); c'est ce mouvement intestin qui, suivant les physiciens dont nous parlons, constitue la chaleur."

Lavoisier et Laplace décident de ne pas trancher. Laplace préfère la seconde conception fondée sur la force vive résultant des mouvements des molécules et s'exprimant par la somme des produits de la masse de chaque molécule par le carré de sa vitesse, alors que Lavoisier restera jusqu'au bout attaché à l'hypothèse d'une substance réelle, "un fluide très subtil qui s'insinue à travers les molécules de tous les corps et qui les écarte". C'est ainsi qu'il explique les états de la matière dans les premières pages de son Traité élémentaire de Chimie, paru en 1789 [7]: les corps "sont ou solides, ou liquides, ou dans l'état élastique et aériforme, suivant le rapport qui existe entre la force attractive de leurs molécules et la force répulsive de la chaleur, ou, ce qui revient au même, suivant le degré de chaleur auquel ils sont exposés." Lavoisier désigne ce "fluide éminemment élastique" qui produit la chaleur sous le nom de calorique, mais prudemment il ajoute: "nous ne sommes pas même obligés de supposer que le calorique soit une matière réelle: il suffit (...) que ce soit une cause répulsive quelconque qui écarte les molécules de la matière, et on peut ainsi en envisager les effets d'une manière abstraite et mathématique." Le concept de calorique sera 
définitivement abandonné plus d'un demi-siècle plus tard [8].

Lavoisier et Laplace étaient néanmoins d'accord sur un principe essentiel commun aux deux hypothèses; ils l'expriment de la façon suivante dans leur Mémoire sur la Chaleur: "Si, dans une combinaison ou dans un changement d'état quelconque, il y a diminution de chaleur libre, cette chaleur reparaîtra tout entière lorsque les substances reviendront à leur premier état, et, réciproquement, si, dans la combinaison ou dans le changement d'état, il y a une augmentation de chaleur libre, cette nouvelle chaleur disparaîtra dans le retour des substances à leur état primitif. (...) Toutes les variations de chaleur, soit réelles, soit apparentes, qu'éprouve un système de corps, en changeant d'état, se reproduisent dans un ordre inverse, lorsque le système repasse à son premier état." En d'autres termes, la chaleur mise en jeu (absorbée ou dégagée) au cours d'une transformation d'un corps (changement d'état, réaction chimique) ne dépend que de l'état initial et de l'état final. Un tel principe n'est pas correct car la chaleur n'est pas en fait une fonction d'état du système. II faudra attendre la seconde moitié du XIXe siècle avec les travaux de Mayer, Thomson et Clausius pour que le premier principe de la thermodynamique soit énoncé avec le concept indispensable d'énergie interne et appliqué correctement à la chimie. On perçoit néanmoins à travers les propos de Lavoisier et Laplace les prémisses de la thermochimie.

\section{CONCEPTION ET RÉALISATION DES CALORIMÈTRES À GLACE}

L'article premier du Mémoire sur la Chaleur [6] comporte une réflexion pertinente sur la façon de déterminer avec précision une quantité de chaleur. Cette réflexion conduit à la conception du modèle de la sphère de glace creuse qui est remarquable: un corps préalablement chauffé à une certaine température et placé à l'intérieur d'une telle sphère rayonne de la chaleur qui est arrêtée par la glace intérieure et sert uniquement à faire fondre de la glace jusqu'à ce que la température du corps atteigne la température de la glace fondante (zéro degré). Le poids de glace fondue est proportionnel à la chaleur perdue par le corps lorsque celui-ci passe de la température initiale à zéro degré; une quantité de chaleur double doit en effet faire fondre deux fois plus de glace. Ainsi, par exemple, la chaleur spécifique d'un corps peut-elle être évaluée en divisant le poids d'eau résultant de la fusion de la glace par le produit de la masse du corps et sa variation de température exprimée en degrés [9].

Ces réflexions sur le modèle de la sphère de glace creuse, évidemment irréalisable pratiquement, ont conduit Lavoisier et Laplace à concevoir un calorimètre à glace [10] fonctionnant selon le même principe: la quantité de chaleur rayonnée par un corps qui se refroidit est
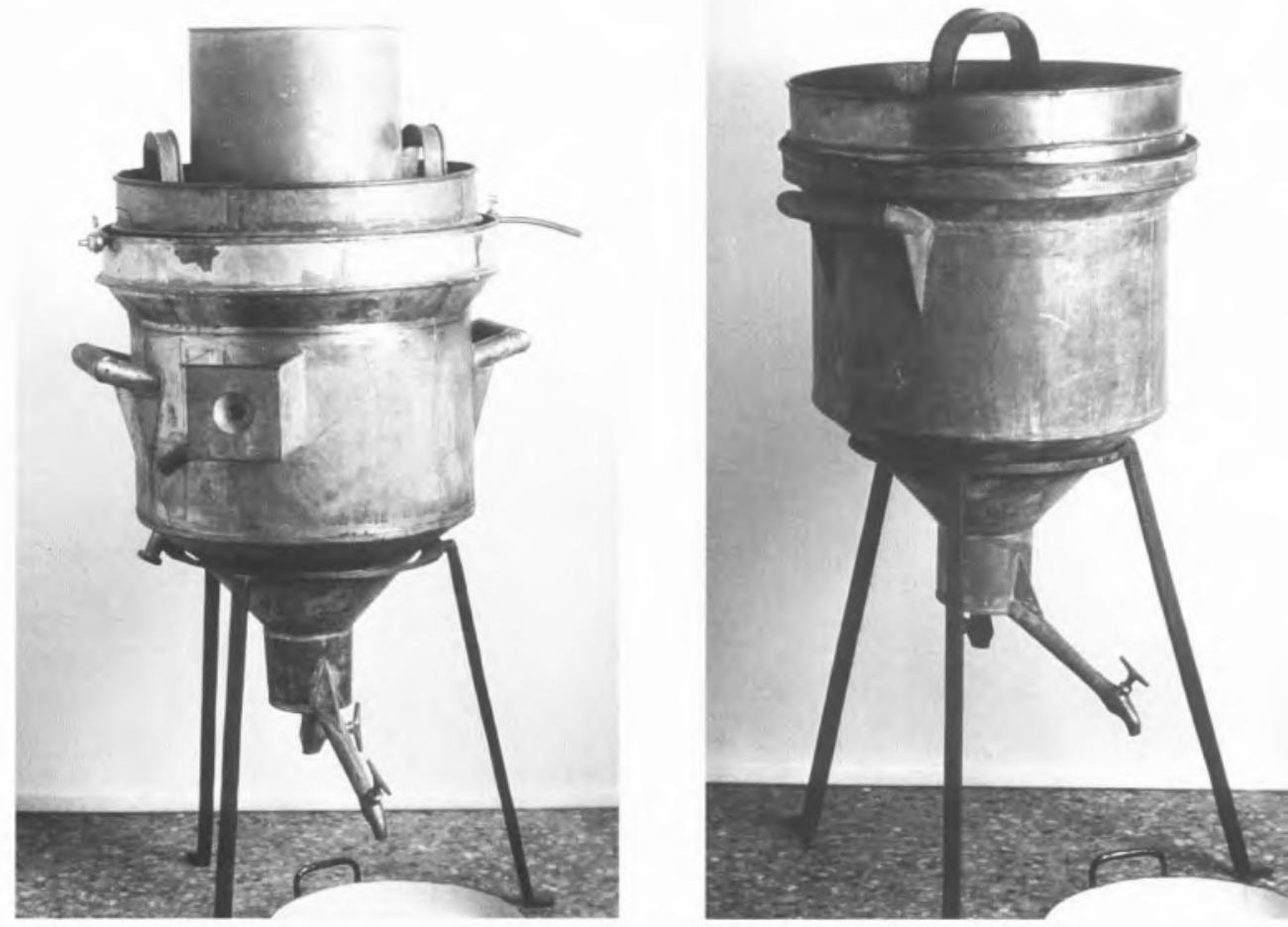

proportionnelle à la quantité de glace fondue sous l'influence de ce rayonnement, et la masse d'eau produite permet de mesurer cette quantité de chaleur.

C'est en 1782 que Lavoisier fait construire deux calorimètres à glace (Figure 1). On ne sait pas de façon certaine qui fabriqua ces calorimètres, mais il s'agit très vraisemblablement du ferblantier Naudin à qui Lavoisier faisait appel au moins depuis 1774; des factures de réparations effectuées par Naudin datant de

Figure 1. Calorimètres à glace de Lavoisier et Laplace. (Photos J.C. Wetzel, (C) Musée des Arts et Métiers-Paris)

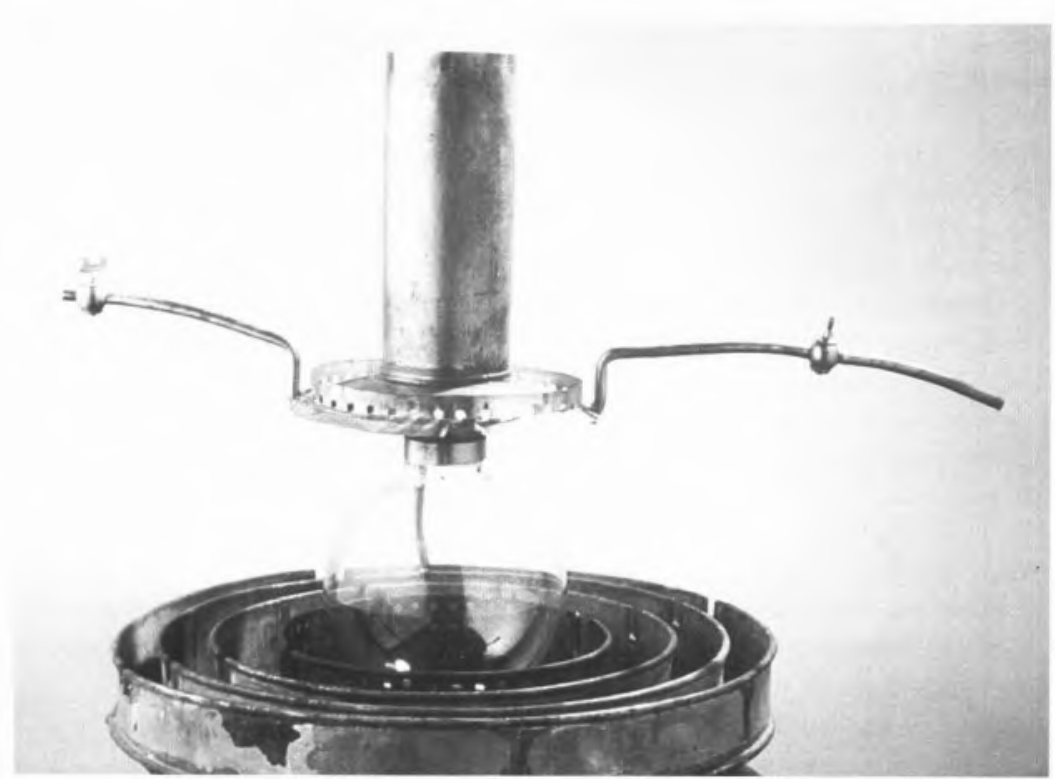



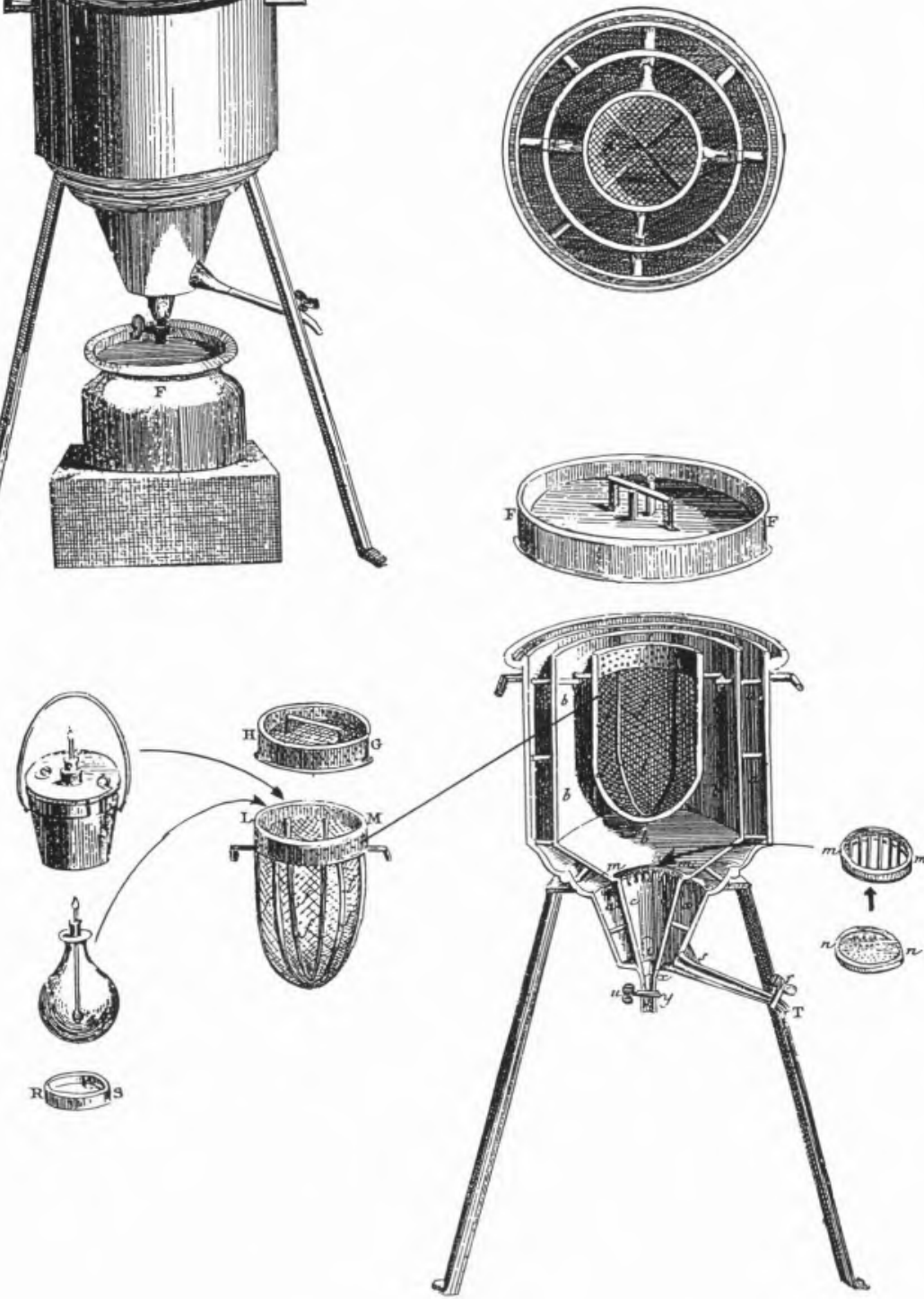

Figure 2. Eléments d'un calorimètre à glace extraits de la planche VI du Traité élémentaire de Chimie [7]. Les éléments de cette planche ont été redisposés et des flèches ont été ajoutées pour des raisons de clarté. Il est intéressant de noter que toutes les planches des ouvrages de Lavoisier ont été dessinées par son épouse Marie-Anne, collaboratrice très active.
1783 ont d'ailleurs été retrouvées [11]. A l'origine, les deux calorimètres étaient identiques puis l'un d'eux sera modifié en 1786 dans le but de mesurer la chaleur de formation de l'eau [12].

La Figure 2 représente les élé- ments d'une planche du Traité élémentaire de Chimie [7] et permet de comprendre le fonctionnement du calorimètre qui est divisé en trois compartiments:

- le compartiment central $L M$ est formé d'un grillage de fil de fer dans lequel on place l'objet de l'expérience; la partie supérieure est fermée par un couvercle $H G$;

- le compartiment intermédiaire est destiné à recevoir de la glace pilée qui est retenue par une grille $\mathrm{mm}$ et un tamis $n n$; la chaleur que dégage l'objet de l'expérience fait fondre la glace et l'eau coule à travers le tamis dans un vase;

- le compartiment externe, également rempli de glace pilée, sert d'isolant: il arrête la chaleur venant de l'extérieur; l'eau résultant de la fusion de la glace est évacuée par un tube $T$.

L'ensemble est recouvert d'un couvercle sur lequel de la glace est déposée, toujours dans un but d'isolation vis à vis de l'atmosphère ambiante.

Une expérience de mesure de la chaleur spécifique se déroule de la façon suivante. Les compartiments intermédiaire et externe ainsi que les couvercles intérieur et extérieur sont remplis de glace pilée bien tassée. Une fois que la glace est bien égouttée, on introduit rapidement dans le compartiment central l'objet de l'expérience préalablement chauffé à une température connue. S'il s'agit d'un corps solide, il est placé dans un seau en tôle muni d'un thermomètre; s'il s'agit d'un liquide, il est disposé dans un matras en verre également muni d'un thermomètre. Une expérience à blanc, c'est à dire avec les récipients vides, est nécessaire pour tenir compte de leur propre chaleur rayonnée. On attend que les corps soient totalement refroidis et on pèse l'eau recueillie dans le vase. L'expérience dure 10 à 20 heures selon les cas.

Ce type de calorimètre très original et astucieux permet non seulement de déterminer la chaleur spécifique des corps mais aussi la chaleur dégagée au cours de réactions chimiques, ou encore par des animaux.

II est évidemment nécessaire de travailler l'hiver pour disposer de glace. Dans leur Mémoire sur la Chaleur, Lavoisier et Laplace décrivent avec rigueur les précautions à prendre. On ne doit pas opérer dans une atmosphère en dessous de zéro degré, sinon la température de la glace ne serait pas de zéro degré mais inférieure. "Ainsi dans un temps de gelée, il faudra renfermer la machine dans un appartement dont on aura soin d'échauffer l'intérieur'. Lorsque la température extérieure est d'une dizaine de degrés au dessus de zéro degré, on ne peut pas empêcher l'air extérieur de pénétrer dans le compartiment intérieur, car l'air situé dans ce compartiment est spécifiquement plus lourd que l'air extérieur; il s'écoule donc par le tuyau $x y$ et il est remplacé par l'air extérieur qui contribue à la fusion de la glace. Pour rendre cette source d'erreur presque négligeable, il est souhaitable d'opérer à des températures extérieures ne dépassant pas 3-4 degrés. "Nous pouvons, à cette température, répondre de l'exactitude de nos 
expériences sur les chaleurs spécifiques des corps, à un quarantième près, et même à un soixantième près, si la température extérieure n'est que de un ou deux degrés."

Pour les expériences nécessitant un renouvellement de l'air (combustion, expérience sur la respiration des animaux), les deux couvercles sont percés de deux trous par lesquels passent deux petits tuyaux permettant d'insuffler de l'air.

\section{RÉSULTATS DES EXPÉRIENCES EFFECTUÉES AVEC LES CALORIMÈTRES À GLACE}

Par malchance, I'hiver 1782-83 est doux. Pourtant Lavoisier et Laplace réussiront à mener à bien de nombreuses expériences. Ils rapportent les chaleurs spécifiques de tous les corps à celle de l'eau prise égale à l'unité. Ils trouvent que "la chaleur nécessaire pour fondre une livre de glace pouvait élever de 60 degrés (Réaumur) la température d'une livre d'eau; (...) il suit de là que la glace absorbe 60 degrés de chaleur en devenant fluide". Le Tableau 1 rassemble les résultats tels qu'ils sont présentés dans le Mémoire sur la Chaleur [6]. Ces tables comportent non seulement des chaleurs spécifiques (ce que Black avait su mesurer auparavant mais avec la méthode des mélanges qui était d'un emploi beaucoup plus limité que le calorimètre à glace), mais surtout des chaleurs dégagées au cours de réactions chimiques qui constituaient donc les premières expériences de thermochimie. On remarquera en particulier la réaction de l'acide sulfurique avec l'acier, les réactions de combustion du charbon, du phosphore ou de l'éther, la détonation du nitre (nitrate de potassium) avec du soufre,... Cette dernière réaction présente un intérêt tout particulier car la poudre à canon était constituée de nitrate de potassium, de soufre et de charbon. N'oublions pas que Lavoisier exerça les fonctions de régisseur des Poudres et Salpêtres.

Autre grande première, la mesure de la chaleur animale: un cochon d'Inde est placé dans un petit panier garni de coton; l'ensemble est introduit dans le calorimètre. Pendant les 5 à 10 heures que dure l'expérience, l'air est renouvelé à plusieurs reprises au moyen d'un soufflet. Les auteurs observent que "l'animal n'a point paru souffrir dans ces expériences"! Cette expérience vise à montrer que la respiration est une combustion lente: la consommation d' "air vital" (oxygène) par l'animal produit de l' "air fixe" (dioxyde de carbone) et fabrique de la chaleur. Lavoisier pense que les poumons sont le siège de la combustion; en fait, la combustion a lieu dans les cellules des tissus, ce qui sera démontré près d'un siècle plus tard.

Durant l'hiver 1783-1784, Lavoisier et Laplace poursuivent leurs expériences sur les chaleurs spécifiques de divers corps et notamment des gaz (air, oxygène,...), sur les chaleurs de combustion (carbone, bougie, huile d'olive,...) et sur la chaleur de respiration des cochons d'Inde et des moineaux.

La dernière expérience, et non des moindres, utilisant un calorimètre à glace a pour objectif la mesure de la chaleur de formation de l'eau. Elle est réalisée durant l'hiver 1785-1786. Avant de décrire cette expérience il est souhaitable de rappeler que de nombreuses expériences avaient été entreprises auparavant sur la combustion de l'hydrogène et de l'oxygène par Lavoisier, Macquer, Warltire et Cavendish. C'est ce dernier qui démontra sans équivoque au printemps de 1783 que cette combustion donne de l'eau, mais il était trop imprégné de la théorie du phlogistique [13] pour conclure que l'eau était constituée d'hydrogène et d'oxygène. Quelques mois plus tard, le 24 juin 1783, Lavoisier, Meusnier, Laplace, Vandermonde, de Fourcroy,... réalisent ensemble l'expérience décisive de la synthèse de l'eau, et dès le lendemain ils annoncent à l'Académie: "L'eau n'est pas une substance simple; elle est composée poids pour poids d'air inflammable (hydrogène) et d'air vital (oxygène)." Le rapport des poids n'est pas correct car les quantités d'oxygène et d'hydrogène n'avaient pas été mesurées à l'aide de gazomètres comme ce fut le cas plus tard. La grande expérience (quantitative) de la synthèse de l'eau a lieu les 27,28 février et 1er mars 1785 en présence de plusieurs savants et des commissaires de l'Académie.

L'hiver suivant, l'un des calorimètres à glace est modifié afin de pouvoir y disposer un ballon muni de deux tubes permettant l'arrivée d'hydrogène et d'oxygène. On ne dispose pas d'une description détaillée de l'expérience, mais le protocole expérimental doit être identique à celui décrit pour la synthèse de l'eau hors calorimètre: après avoir fait le vide dans le ballon, on le remplit

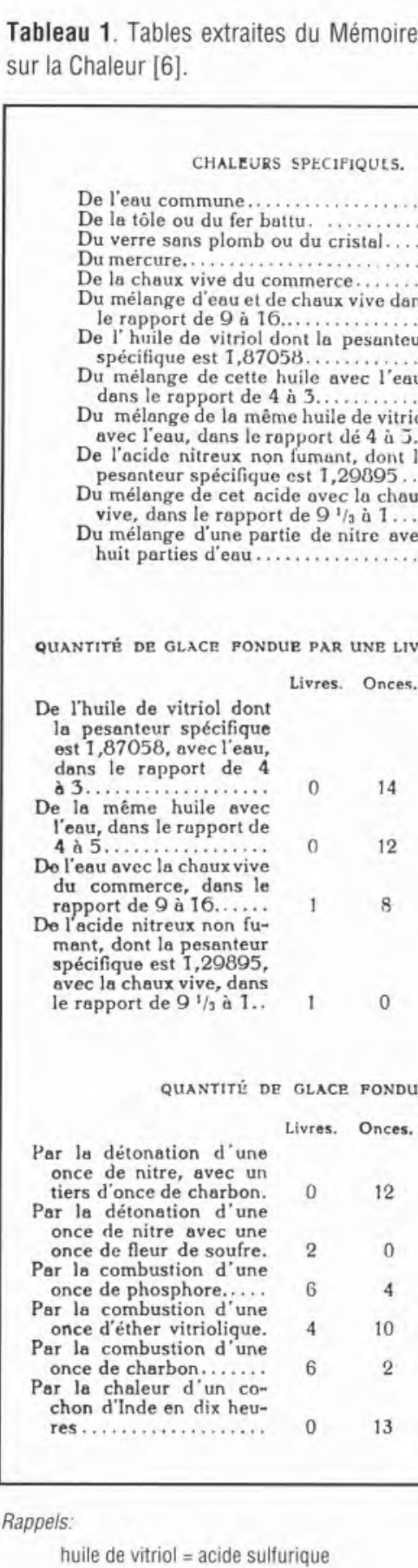

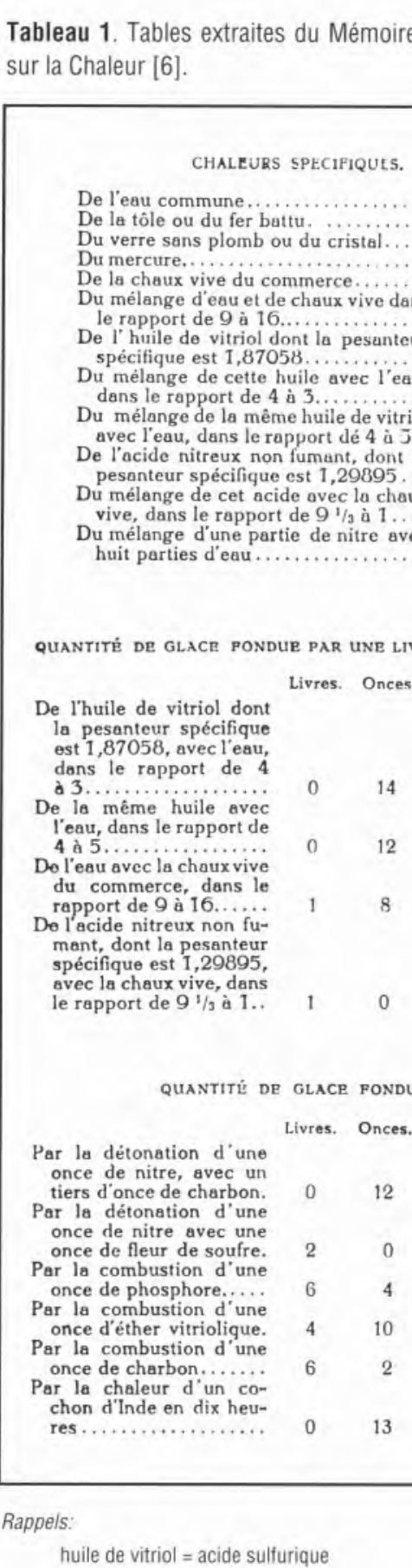

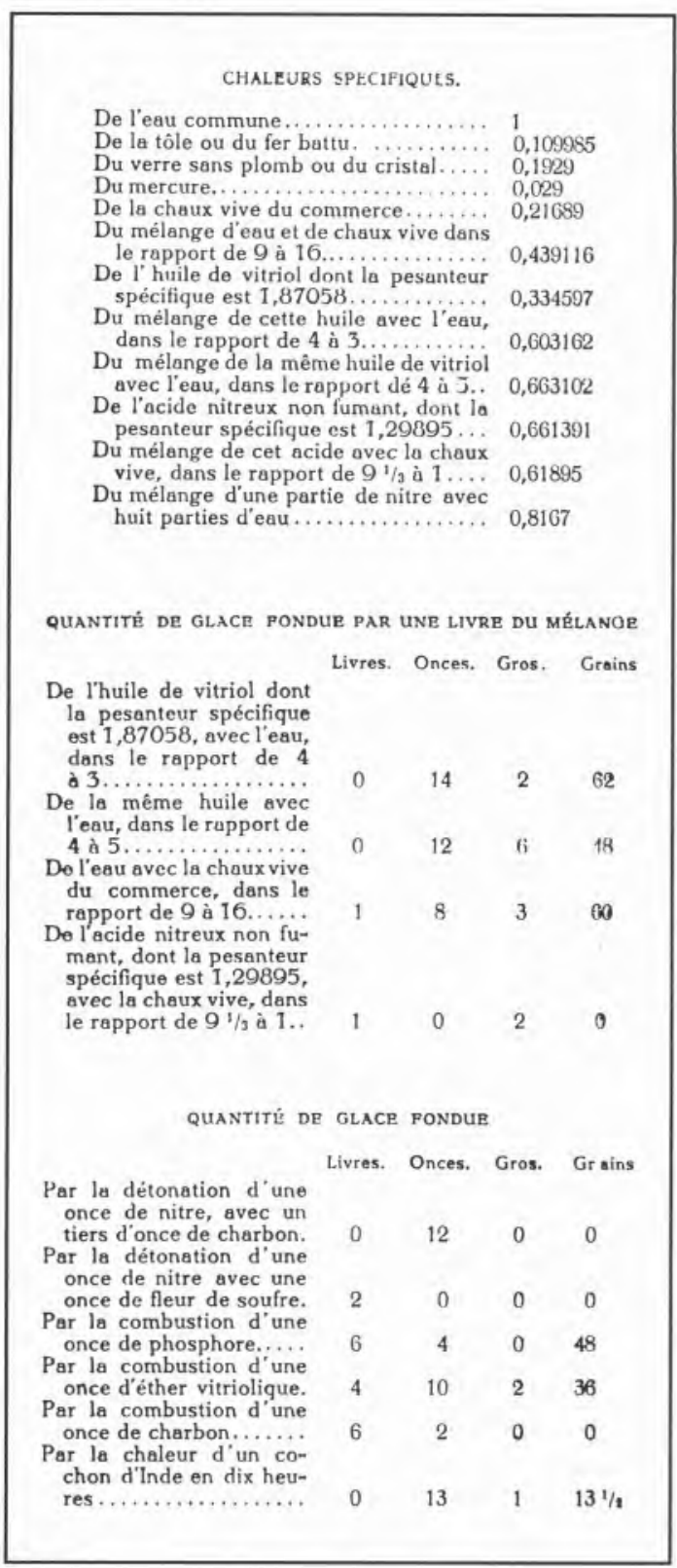

Rappels:

nitre $=$ nitrate de potassium

1 livre $=489,51$ grammes

1 once $=30,59$ grammes

1 gros $=3,824$ grammes

1 grain $=0,053$ grammes

d'oxygène; puis l'hydrogène arrivant par un tube plongeant dans celui-ci est enflammé par un dispositif fournissant une étincelle électrique. L'hydrogène et l'oxygène sont issus de gazomètres qui permettent de connaître les quantités 
Tableau 2. Table extrait du Traité élémentaire de Chimie [7].

\section{Combuftion du Gaz hydrogène.}

Quantité de gaz hydrogène brûlé, liv. onc. gros gr.

Quantité de gaz oxygène employé

pour la combultion,

Quantité d'eau formée,

Quantité de calorique dégagé par la combuftion d'une livre de gaz hydrogène, 295,58950

Quantité de calorique dégagé par chaque livre de gaz oxygène,

Quantité de calorique qui fe dégage pendant la formation d'une livre d'eau ,

Quantité de calorique que conferve une livre d'oxygène dans fa combuftion avec l'hydrogène,

Quantité de calorique que conferve une livre d'eau à zéro,
44,33840

14,50386

12,32823 utilisées. Les résultats sont exposés dans le Traité élémentaire de Chimie [7] (Tableau 2). La quantité de calorique est exprimée en livres de glace fondue et rapportée à 1 livre d'hydrogène brûlé. Le nombre de chiffres avec lequel le résultat est donné paraît excessif : 295,58950; et la précision sur la quantité d'oxygène consommé et la quantité d'eau formée est quelque peu surprenante quand on sait qu'1 grain représente 53 milligrammes ! Laissons de côté ces interrogations pour ne retenir que le fait historique: pour la première fois la chaleur de formation d'un composé à partir de ses éléments a pu être déterminée.

\section{CONCLUSION}

Ainsi l'histoire des calorimètres à glace est exemplaire à divers titres. Tout d'abord, il faut souligner la démarche scientifique de Lavoisier et Laplace qui est fondée sur - l'analyse des phénomènes à étudier, - la conception d'un appareil susceptible de donner des résultats précis et fiables, l'étude rigoureuse des sources d'erreur,

- la réalisation d'expériences avec le souci de concordance des mesures, - enfin l'analyse des résultats avec esprit critique.

Cette démarche est universelle et ces travaux fournissent donc un des exemples les plus remarquables de l'histoire des sciences tant par la méthodologie que par la réalisation et les résultats. La pensée et la méthodologie de Lavoisier s'inscrivent dans l'évolution intellectuelle et scientifique du XVIIIe siècle, le "Siècle des lumières". Ce n'est pas le pourquoi des choses, mais le comment qui importe; les faits doivent être contrôlés et, sous-jacente, la notion optimiste de progrès sous-tend la démarche rationnelle.

On peut noter également le souci de réserve vis à vis de concepts nouveaux comme le calorique, introduit par commodité comme une hypothèse mais de façon non dogmatique. Cette hypothèse de l'existence d'un fluide n'a pas résisté pas à l'épreuve des faits par la suite, mais elle n'a pas freiné l'avancement de la connaissance, au contraire. Ce n'est pas le seul exemple de l'histoire des sciences où un concept constitue temporairement un outil pour expliquer des phénomènes physiques ou chimiques, puis il doit être modifié ou abandonné à la suite de nouveaux faits expérimentaux dont il ne peut rendre compte. II faut d'ailleurs reconnaître que le concept de chaleur est particulièrement difficile à décrire.

Un autre point qui mérite d'être souligné et qui est une particularité habituelle des travaux conduits par Lavoisier, c'est la mise en oeuvre du travail en équipe. A son initiative, Lavoisier a entrepris des travaux sur la chaleur avec Laplace. Ces travaux touchant à la physique, la chimie et même la biologie, la complémentarité de leurs compétences s'est avérée féconde. En outre, les discussions approfondies, la confrontation des idées, la critique objective conjointe des résultats sont certainement autant d'éléments clés de la réussite de leurs recherches. Quel exemple pour les générations suivantes!

\section{RÉFÉRENCES ET NOTES}

1. J.-P. Poirier, Lavoisier, Ed. Pygmalion-Gérard Watelet, Paris, 1993.

2. B. Bensaude-Vincent, Lavoisier, Mémoire d'une révolution, Ed. Flammarion, Paris, 1993.

3. L'Actualité Chimique, mars-avril 1994, Ed Dunod, Paris (12 articles).

4. La Revue, mars 1994, Ed. Musée National des Techniques, Paris (7 articles).

5. Química, Boletim da Sociedade Portuguesa de Quimica, n 52, 1994 (2 articles); n 53, 1994 (1 article).

6. A.-L. Lavoisier et P.-S. Laplace, Mémoire sur la Chaleur, réédition Gauthier-Villars, 1920. La publication originale figure dans les Mémoires de I'Académie des Sciences, année 1780, p. 355 , bien que la lecture en fut donnée les 18 et 25 juin 1783 à l'Académie.

7. A.-L. Lavoisier, Traité élementaire de Chimie, 1789. Réimpression de l'édition originale, Ed. J. Gabay, Paris, 1992.

8. P. Brouzeng et D. Fauque: Eléments d'histoire d'un concept disparu: le calorique, L'Actualité Chimique, Mars-Avril 1994, p. 32-35.

9. Dans le Mémoire sur la Chaleur [6], l'échelle Réaumur $\left(0^{\circ}\right.$ pour la glace fondante, $80^{\circ}$ pour l'eau bouillante) est utilisée.

10. Dans le Mémoire sur la Chaleur [6], le nom de machine est utilisé; c'est dans le Traité élémentaire de Chimie [7] que le nom de calorimètre sera utilisẻ pour la première fois. Lavoisier est conscient que ce mot vient en partie du latin et en partie du grec et il le justifie ainsi: "mais j'ai cru qu'en matière de science on pouvait se permettre moins de pureté dans le langage, pour obtenir plus de clarté dans les idées".

11. M. Daumas: Lavoisier, théoricien et expérimentateur, Presses Universitaires de France, Paris, 1955.

12. Ces deux calorimètres font partie de la riche collection des instruments de Lavoisier que possède le Musée National des Techniques au Conservatoire National des Arts et Métiers à Paris.

13. Dans la théorie du phlogistique élaborée par Stahl, tous les corps combustibles étaient considérés comme formés d'un constituant non combustible uni à un fluide inflammable que l'on appelait le phlogistique.

*Laboratoire de Chimie Générale Conservatoire National des Arts et Métiers, Paris 
Exmos. Senhores,

Querendo acompanhar as necessidades dos seus clientes, a SOQUIMICA criou o laboratório de ensaios SOQUILAB que está integrado no Sistema Português da Qualidade, conforme nossa comunicação de 1 de Outubro de 1993.

É com satisfação que comunicamos que,

\section{O SOQUILAB É UM LABORATÓRIO ACREDITADO PELO IPQ}

Esta certificação foi concedida pelo IPQ para a calibração de instrumentos de pesagem não automáticos de equilibrio automático e semi-automático e agora para a calibração de espectrofotometros, tituladores, buretas, condutimetros e aparelhos de $\mathrm{pH}$.

\section{A SOQUIMICA É A PRIMEIRA EMPRESA PRIVADA ACREDITADA PARA A CALIBRAÇÃO DE BALANÇAS, ESPECTROFOTOMETROS, TITULADORES, BURETAS, CONDUTIMETROS E APARELHOS DE $\mathrm{pH}$}

Foi já iniciada a terceira fase deste processo, estando pronta a extensão do dominio da calibração a outras áreas e equipamentos.

Para mais informações podem V. Exas. contactar o director do SOQUILAB, Eng ${ }^{\circ}$ José $^{-}$ António Reis, utilizando os mesmos endereços e telefones da SOQUIMICA.

Esperando que este serviço cumpra as necessidades e expectativas de V. Exas, subscrevemo-nos com elevada estima e consideração,

De V. Exas.

Atentamente

SOQUIMICA

Soc. de Representações de Química, Lda.

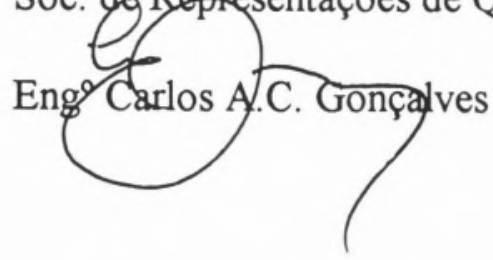

